\title{
ANALYSIS AND CORRELATION OF IMAGING FINDINGS WITH THE ANATOMOPATHOLOGICAL STUDY OF BI-RADS 4A LESIONS
}

Thais Bianco', Renata Drizlionoks¹, Gabrielle Fernandes de Paula Castanho¹, Gabriel Lucca de Oliveira Salvador ${ }^{1}$, Maria Helena Louveira'

'Hospital das Clínicas, Universidade Federal do Paraná - Curitiba (PR), Brazil.

Introduction: The Breast Imaging Reporting and Data System (BI-RADS ${ }^{\circledR}$ ) was developed in 1993 by the American College of Radiology to standardize reports based on imaging findings, classifying them into six categories. Its fourth edition proposed the subdivision of category 4 into three subcategories according to malignancy suspicion. Category 4A shows likelihood of malignancy between $2 \%$ and $10 \%$, and diagnosis by biopsy is recommended. Frequent histological findings in the literature for 4A lesions include fibrocystic breast changes, fibroadenoma, columnar cell lesions with atypia, stromal sclerosis, inflammatory disorders, and proliferative epithelial lesions. Objectives: To show the most relevant radiological and histological findings for the BI-RADS ${ }^{\circledR} 4 \mathrm{~A}$ subcategory, corroborating its likelihood ratio of malignancy. Methods: This is a cross-sectional study based on the review of medical records of patients submitted to the anatomopathological study of BI-RADS 4A lesions in public and private health services from Curitiba, Paraná, Southern Brazil, between March and September 2019. The findings were subsequently correlated with histopathological results. Results: A total of 727 core needle breast biopsies were performed $-78.6 \%$ guided by ultrasound and $21.4 \%$ by stereotaxy. Approximately $35.8 \%$ of ultrasound-guided procedures (group X) and 55.4\% of stereotaxic biopsies (group Y) were classified as BI-RADS 4A. Among the main imaging findings in group X, solid nodules, solid cystic lesions, and solid heterogeneous areas stood out. Group Y presented clusters of heterogeneous, punctate, amorphous microcalcifications, and findings that did not fit the BI-RADS classification. Benign changes predominated among the histopathological findings in both groups. The malignancy rate according to guidelines of the European classification for anatomopathological results of breast lesions 4 remained around $2 \%$ in group X and $8.7 \%$ in group Y. Conclusions: Based on the results obtained, we concluded that the malignancy rates of biopsies from patients classified as BI-RADS 4A were within the acceptable values established by the literature. However, they varied considerably according to the biopsy method chosen, presenting higher values in patients submitted to stereotaxy. 